\title{
Interleukin-11 plays a key role in human and mouse alcohol-related liver
} disease

Effenberger Maria ${ }^{1}$, Grabherr Felix ${ }^{1}$, Schaefer Benedikt ${ }^{1}$, Grander Christoph ${ }^{1}$, Mayr Lisa1, Schwärzler Julian', Enrich Barbara¹, Moser Patrizia², Fink Julia1, Pedrini Alisa1, Jaschke Nikolai ${ }^{1}$, Freund Martin ${ }^{3}$, Loizides Alexander ${ }^{3}$, Bale Reto $^{3}$, Putzer Daniel $^{3}$, Widjaja Anissa $A^{4}$, Schafer Sebastian ${ }^{4,5}$, Cook Stuart $A^{4,5,6}$, Zoller Heinz ${ }^{1}$, Oberhuber Georg $^{2}$, Adolph Timon E1, Tilg Herbert ${ }^{1}$

${ }^{1}$ Department of Internal Medicine I, Gastroenterology, Hepatology, Endocrinology and Metabolism, Medical University of Innsbruck, Innsbruck, Austria 2 INNPATH, Institute of Pathology, University Hospital of Innsbruck, Innsbruck, Austria

${ }^{3}$ Department of Radiology, Medical University of Innsbruck, Innsbruck, Austria

${ }^{4}$ Cardiovascular and Metabolic Disorders Program, Duke-National University of Singapore Medical School, Singapore, 169857, Singapore

${ }^{5}$ National Heart Research Institute Singapore, National Heart Centre Singapore, Singapore, 169609, Singapore

${ }^{6} \mathrm{MRC}$-London Institute of Medical Sciences, Hammersmith Hospital Campus, London, W12 ONN, UK

Corresponding author:

Herbert Tilg, MD

E-mail: herbert.tilg@i-med.ac.at

Anichstrasse 35

A-6020 Innsbruck 


\begin{abstract}
Background: Alcoholic hepatitis $(\mathrm{AH})$ reflects acute exacerbation of alcoholic liver disease (ALD) and is a growing healthcare burden worldwide with limited treatment options. Interleukin-11 (IL-11) is a pro-fibrotic, pro-inflammatory cytokine with increasingly recognized toxicities in parenchymal and epithelial cells.
\end{abstract}

Aim: The aim of this study was to explore the prognostic value of IL-11 serum levels in patients suffering from $\mathrm{AH}$ and cirrhosis of various etiology and to understand the role of IL-11 in experimental ALD.

Methods: IL-11 serum concentration and tissue expression was determined in a cohort comprising 50 patients with $\mathrm{AH}, 110$ patients with cirrhosis and 19 healthy volunteers. Findings were replicated in an independent patient cohort including 186 patients. Ethanol-fed wildtype mice were treated with a neutralizing murine IL-11 receptorantibody (anit-IL11RA) and thereafter examined for severity signs and markers of ALD.

Results: Human IL-11 serum concentration and liver tissue expression increased with severity of liver disease and were most pronounced in $\mathrm{AH}$. In a multivariate Coxregression, a serum level above 6.4 picograms/milliliter was a MELD independent risk factor for transplant-free liver disease survival in patients with compensated and decompensated cirrhosis. Findings were confirmed in an independent cohort. In mice, severity of alcohol-induced liver inflammation was positively correlated to enhanced hepatic IL-11 expression. Pretreatment with a neutralizing anti-IL11RA inhibited hepatic inflammation and mice were protected from ethanol-induced liver injury. In comparison to IgG-control, ethanol-fed mice treated with anti-IL11RA showed 
decreased steatosis, hepatic neutrophil infiltration, and expression of pro-inflammatory cytokines.

Conclusion: IL-11 plays a crucial role in the pathogenesis of ALD and could serve as an independent prognostic factor for transplant-free survival. Blocking IL-11 signaling might be a therapeutic option in human ALD, particularly $A H$. 


\section{Introduction}

Chronic alcohol consumption causes physical and psychiatric disabilities and contributes to approximately 3 million deaths each year worldwide. Overall, ethanol abuse is responsible for $5.1 \%$ of the global burden of disease ${ }^{1}$. Alcoholic liver disease (ALD) is a common liver disease and is a complex pro-inflammatory process leading to steatosis, alcoholic hepatitis $(\mathrm{AH})$, fibrosis, cirrhosis, and finally hepatocellular carcinoma ${ }^{2}$. The pathogenesis of $A L D$, especially $A H$, is poorly understood which is reflected by poor therapeutic options and patient outcome. Along with direct toxicity of ethanol and acetaldehyde, alcohol causes gut dysbiosis, which subsequently provokes an altered gut barrier function and endotoxemia ${ }^{3-5}$. This dysregulation also induces expression of various pro-inflammatory cytokines in the liver which contribute to cell death and liver injury 6 . Interleukin (IL)-6, tumor necrosis factor- $\alpha$ (TNF- $\alpha$ ), and IL-1 $\beta$ are all important for ALD in experimental models ${ }^{7-9}$.

$\mathrm{IL}-11$ is one of eleven known IL-6 family members ${ }^{10,11}$. It is secreted from epithelial cells and hepatocytes in response to injury and from activated fibroblasts, acting in an autocrine and paracrine manner. In epithelial cells and hepatocytes, IL-11 causes cellular dysfunction and can initiate cell death processes while blocking regeneration ${ }^{12,13}$. In stromal cells, IL-11 triggers extracellular matrix production and invasion and migration of myofibroblasts ${ }^{14}$. To complete this vicious cycle, activated fibroblasts and myofibroblasts then secrete pro-inflammatory cytokines and chemokines ${ }^{15}$. All members of the IL-6 family require the ubiquitously expressed gp130 receptor to activate downstream signaling pathways. IL-11 was often compared to IL6 , as both cytokines form a similarly arranged gp130 heterodimer complex to initiate cell activation. Interestingly, the a-subunit receptors of these two cytokines are 
expressed in almost mutually exclusive cell types and IL-11 and IL-6 are proving to have very different functions ${ }^{16}$.

Little is known about the role of IL-11 in liver disease and its impact on inflammation of the liver. Recently published experiments showed a central role for endogenous and species-matched IL-11 in the pathology of fibro-inflammatory diseases, such as inflammatory bowel disease, myocardial and lung fibrosis, systemic sclerosis, and, most importantly, non-alcoholic liver disease where direct hepatotoxic effects have been shown ${ }^{16-19}$.

We investigated circulating $\mathrm{IL}-11$ and its expression in the liver in a patient cohort suffering from cirrhosis and $\mathrm{AH}$ and establish the concept of $\mathrm{IL}-11$ signaling as driver of experimental ALD. 


\section{Materials and Methods}

\section{Patients}

Patient's samples of the training cohort $(n=179)$ were collected at the University Hospital of Innsbruck between 2017 and 2019, whereas samples of the validation cohort $(n=186)$ were collected between 2019 and 2020.

Cirrhosis was confirmed by abdominal computed tomography and by indirect cirrhosis signs, such as esophageal varices, portal hypertension, ascites, hepatic encephalopathy, or thrombocytopenia. The Model of End stage Liver Disease (MELD) score was calculated. Distribution of etiology of liver disease in the training group ( $n=160)$ was as follows: 79 patients had compensated cirrhosis and 31 patients had decompensated cirrhosis. The underlying etiology of liver disease is summarized in Table 1. Decompensated cirrhosis was defined by presence of ascites, bleeding, encephalopathy, or jaundice ${ }^{20}$. Patients diagnosed with cirrhosis due to alcoholic fatty liver disease and confirmed alcohol abstinence $>6$ months were included in the cirrhosis group. Another group in the training cohort consisted of patients with alcoholic hepatitis $(A H)(n=50)$ which was diagnosed upon an acute onset of jaundice in the past 8 weeks arising on the background of severe alcohol abuse. The thresholds was defined at an average alcohol consumption of more than 3 drinks (40 grams $(\mathrm{g})$ )/day for women and 4 drinks (50-60 g)/day for men. Patients diagnosed with $\mathrm{AH}$ were addicted for at least 6 months with no abstinence until 60 days before the onset of jaundice. The diagnosis of $\mathrm{AH}$ required a serum bilirubin $>3 \mathrm{mg} / \mathrm{dl}$ and serum aspartate transaminase $(A S T)>50 \mathrm{IU} / \mathrm{ml}$. It's ratio to alanine aminotransferase $(A L T)$ had to be above 1.5. Patients with AST and ALT levels > $400 \mathrm{U} / \mathrm{ml}$ were excluded from the alcoholic hepatitis group ${ }^{21}$. 
Further exclusion criteria were uncontrolled infection, multiorgan failure, uncontrolled upper gastrointestinal bleeding, preexisting kidney disease with serum creatinine $>2.5$ $\mathrm{mg} / \mathrm{dl}$, hepatocellular carcinoma (HCC) or other active malignancies, pregnant and lactating people and active drug abuse. In the $\mathrm{AH}$ group patients with hepatitis $\mathrm{B}$ or $\mathrm{C}$, acquired human immune deficiency syndrome, autoimmune liver disease, primary biliary cholangitis and primary sclerosing cholangitis, Wilson disease, hemochromatosis, and suspected drug-induced liver injury were excluded.

Healthy volunteers, with no underlying disease, $(n=19)$ were included in our outpatient clinic during regularly check-up $(n=15)$. 4 healthy volunteers underwent liver biopsy as potential living liver donors, no liver disease was detected histologically.

Patients included in the validation cohort $(n=186)$ distributed as followed: 23 healthy volunteers, 85 patients suffering from compensated cirrhosis, 42 patients suffering from decompensated cirrhosis and 36 patients with $\mathrm{AH}$ contributed to the validation patient cohort. Further details of the patient cohort are presented in Table 2.

\section{Ethical consideration}

The study protocol was approved by the institutional ethics commission with an amendment to AN2017-0016 369/4.21.

\section{Mouse studies}

C57BL/6 mice purchased from Charles River Laboratories (Wilmington, MA) were cohoused in the Animal Facility of Medical University of Innsbruck for one week prior to the start of experiments. All mice were fed the Lieber DeCarli pair-fed diet for five days to become acclimated to a liquid diet. Female wild-type (wt) mice (7-8 weeks old) were then fed with a Lieber-DeCarli diet (BioServ, Flemington, NJ) containing an 
increasing amount of ethanol (EtOH) ranging from 1 to 5 vol\% ad libitum for 15 days $(\mathrm{EtOH}-\mathrm{fed})^{22}$. Control diet was supplemented with an isocaloric amount of maltose (pair-fed). Pair-fed mice were calorie matched with the ethanol-fed mice. Mice were weighed every other day. 8 hours after gavage the mice were euthanized. All mice received Xylain $5 \mathrm{mg} / \mathrm{kg}$ bodyweight (Intervet, Vienna, Austria) and Ketamin $100 \mathrm{mg} / \mathrm{kg}$ bodyweight (AniMedica, Senden, Germany) for anesthesia. Blood, tissue samples of liver and intestine were collected afterwards. Serum was stored at $-80^{\circ} \mathrm{C}$ as well as tissue samples were stored at $-80^{\circ} \mathrm{C}$ or in RNAlater (Qiagen, Hilden, Germany) at $-20^{\circ} \mathrm{C}$.

In Vivo administration of anti-IL11RA

Mice were injected intraperitoneally with either anti-IL11RA (kindly provided by the Cook laboratory, Duke-NUS, Singapore) or an identical amount of Immunoglobulin G (lgG) control $(20 \mathrm{mg} / \mathrm{kg}$ ) (kindly provided by the Cook laboratory, Duke-NUS, Singapore). These antibodies have been described in detail in previous publications $^{18,23}$

\section{Ethical considerations}

All anti-IL11RA experiments adhered to ethical principles according to Austrian law (BMWFW-2020.0.547.764) and the ARRIVE Guidelines ${ }^{24}$. The experiments were carried out at the animal facility of the Medical University of Innsbruck.

\section{Data analysis}

Data are expressed as mean \pm standard error of mean or as median with first and third quartiles. For comparing quantitative variables, the Student's t-test or the nonparametric Mann-Whitney U or Wilcoxon signed-rank test were used as appropriate. 
Normality of distribution was determined by Kolmogorov-Smirnov test. The correlation analysis was estimated using the Spearman's p coefficient. Survival analysis was performed using the Kaplan-Meier method applying the log-rank test for group comparison. Kruskal-Wallis test or ANOVA was used where applicable. To identify predictors of mortality, a Cox proportional hazards model was applied. Correlation was carried out using the Spearman's rank correlation coefficient; - to correct for multiple testing, the Benjamin-Hochberg method was used with an accepted false discovery rate of $<0.05$. A p-value $<0.05$ was considered as statistically significant. All statistical analyses were performed using SPSS Statistics v.22 (IBM, Chicago, IL), R Programming and GraphPad PRISM 5 (La Jolla, CA).

Further information on materials and methods are provided in the supplementary material and methods section. 


\section{Results}

Our training cohort included 160 patients with liver disease being treated in-hospital or in our outpatient clinic, with etiologies listed in Table 1. The cohort comprised 79 patients with compensated cirrhosis and 31 patients with decompensated cirrhosis of varying etiology, while 50 patients participated with $\mathrm{AH}$. The study design is depicted in Fig. 1A. To exclude any preexisting liver diseases in patients suffering from alcoholic hepatitis different staining methods have been used, e.g. Masson's trichrome staining or periodic acid shift with diastase staining (Supplementary Fig. 1). Healthy volunteers $(n=19)$ served as control group, with characteristics provided in Table 1.

Severity of ALD correlates with circulating IL-11 levels and its hepatic expression

The MELD score positively correlated with IL-11 levels $\left(p<0.001, r^{2}=0.73\right.$, Fig. 1B) and IL-11 was significantly elevated in cirrhosis and $\mathrm{AH}$ patients compared to healthy controls, respectively. Highest levels were seen in alcoholic hepatitis (Fig. 1C). IL-11 serum levels were higher in decompensated cirrhosis compared to compensated patients (Fig. 1D). There was no correlation of IL-11 levels with C-reactive protein, albumin, INR, liver transaminases, gamma-glutamyl transferase, alkaline phosphatase, leukocytes, and serum creatinine (Supplementary Fig. 2). Interestingly, etiology of liver cirrhosis, excluding $\mathrm{AH}$, was not reflected in different IL-11 levels (data not shown).

To examine IL-11 levels at the level of liver tissue, hematoxylin and eosin (HE) and immunohistochemistry (IHC) IL-11 staining was performed in liver biopsies from healthy volunteers, patients with cirrhosis and alcoholic hepatitis (Fig. 1E). A large 
number of IL-11 positive hepatocytes were detected in ongoing $\mathrm{AH}$, whereas biopsies of cirrhotic liver tissue stained less for IL-11 expression (Fig. 1F).

\section{Serum IL-11 concentration predicts patient outcome}

Univariate and multivariate Cox regression analyses were performed to uncover noninvasive prediction markers for survival up to 24 months without liver transplantation. After Cox regression analyses, non-significant factors like age or gender and factors already represented in the MELD analysis were excluded based on stepwise model selection. Finally, MELD and IL-11 concentration remained in our multivariate model and both turned out to independently predict survival without transplantation for 6,12 , 18, and 24 months (Table 2). The accuracy of IL-11 levels in predicting transplant-free survival was comparable to the established MELD score in a receiver operating characteristics (ROC) analysis (Fig. 2A). Youden's index was calculated to determine an optimal cut-off value for IL-11 for liver disease. IL-11 below $6.4 \mathrm{pg} / \mathrm{ml}$ predicted a transplant-free 6 months survival with a sensitivity of $59.4 \%$ and a specificity of $93.3 \%$ (Fig. 2A). To further evaluate the performance of IL-11 levels, we analyzed the role of IL-11 serum levels in a training cohort $(n=179)$ using the Kaplan-Meier method ( $p$-value of 0.0001$)$ (Fig. 2B). Data were checked and compared in a validation cohort $(n=186)$ (Fig. 2C). Characteristics of the validation cohort are summarized in Supplementary Table 2. Notably, transplant-free survival was significantly lower in the validation cohort (validation cohort vs. training cohort $28 \% ; \mathrm{p}<0.001$ ). Similar as in the training cohort, the accuracy of IL-11 to predict transplant free survival was non - inferior to that of MELD (Supplementary Figure 3). Furthermore IL-11 AUROCs in the training and validation cohort didn't show significant differences (Supplementary Figure 3). Differences between the AUROCs in the training and in the validation cohort were not statistically significant (Supplementary Table 3). 
Inhibition of IL-11 signaling protects against experimental ALD

Female C57BL/6J mice were exposed to a 5\% ethanol containing Lieber-DeCarli diet or an isocaloric pair diet for 15 days. In this experimental set-up EtOH-fed mice received a neutralizing $\mathrm{IL}-11$ receptor antibody (anti-IL11RA) or IgG control intraperitoneally as illustrated in Fig. 3A. Control-treated EtOH-fed mice showed signs of liver injury which was reversed by anti-IL11RA administration (Fig. 3B). Furthermore, anti-IL11RA administration resulted in reduced liver-body ratio compared to IgG control (Fig. 3C) and anti-IL11RA could also prevent the EtOH-induced weight loss (Fig. 3D). The anti-IL11RA effect applied also to $\mathrm{EtOH}$-induced hepatic triglyceride accumulation (Fig. 3E).

\section{Inhibition of IL-11 signaling protects against ethanol-induced liver inflammation}

To investigate any further potential beneficial effects of the anti-IL11RA, we stained hepatic tissue of these mice for IL-11 (Fig. 4A) showing significant lower expression of IL-11 in anti-IL11RA-treated compared to ethanol-fed control mice (Fig. 4B). Since IL11 might induce other pro-inflammatory mediators in the liver we further investigated hepatic gene expression of TNFa, tissue inhibitor of metalloproteinases 1 (TIMP-1), IL10, CXCL1, IL-1ß, macrophage inflammatory proteins (MIP)-2 and IL-6. Anti-IL11RA treatment significantly reduced expression of each of them apart from IL-6, which has been reported to be potentially beneficial in liver disease ${ }^{25}$ (Figure $4 C-4 I$ ). Remarkably, the expression of IL-1 $\beta$ was suppressed below baseline. Additional inflammation and fibrosis marker are shown in Supplementary Fig. 4. 
Anti-IL11RA treatment reduces infiltration of pro-inflammatory cells into the liver After HE-staining of liver tissue 20 HPFs were analyzed, and a steatosis score was calculated. Steatosis score was significantly reduced upon treatment with anti-IL11RA compared to control animals (Fig. 5AB). Myeloperoxidase (MPO+) staining showed an almost complete inhibition of neutrophil immigration into liver tissue by systemic antiIL11RA treatment (Fig. 5CD). This was consistent with decreased F4/80 positive macrophages in ethanol-fed mice treated with anti-IL11RA (Fig. 5EF). 


\section{Discussion}

We herein report increased IL-11 levels in peripheral blood along with upregulated intrahepatic IL-11 expression in patients suffering from advanced liver diseases, specifically in patients with $\mathrm{AH}$. A positive correlation was found with transplant-free survival and a cut off value for IL-11 serum levels was determined and validated in a separate cohort. We assumed IL-11 might play an important role in the hepatotoxicity of ethanol and/or pro-inflammatory processes and that blocking $\mathrm{IL}-11$ receptor may have beneficial effects on multiple components of ALD. This hypothesis is supported by our murine ALD model using a neutralizing IL-11 receptor antibody.

Parenchymal infiltration of neutrophils and macrophages is a prominent feature of ALD and is likely due to ethanol-mediated activation of innate immunity and subsequent induction of pro-inflammatory cytokines and chemokines ${ }^{26-29}$. Recent studies discovered an unexpected pro-inflammatory role for IL-11 in NAFLD and showed that HSCs highly express IL-11 receptor and show an indirect effect of IL-11 on immune cells that is mediated via the stroma ${ }^{18}$. It is also the case that injured hepatocytes, which also highly express IL11RA, secrete IL-11 and this activates HSCs and inflammatory cells in a paracrine manner while damaging hepatocytes in an autocrine fashion ${ }^{13}$. We could corroborate these findings in our study in the context of ALD. In line with this, anti-IL11RA robustly reduced inflammation in our ALD model. Hepatocytes express the IL-11 receptor and secrete cytokines upon ligation, such as transforming growth factor beta $(\mathrm{TGF}-\beta)^{18}$. IL-11 activation of hepatocytes is unexpectedly cytotoxic and an autocrine and maladaptive loop of IL-11 activity in hepatocytes is apparent. The effects of IL-11 on these cell type during acute necroinflammation in non-alcoholic steatohepatitis (NASH) are profound ${ }^{13}$ and were 
also observed in our ALD model. IL-11 blockade was also associated with lower liver fat and reduced hepatic oxidative stress in murine NASH models ${ }^{30}$.

However, former studies have suggested that IL-11 might act cytoprotective, antifibrotic, and anti-inflammatory based on effects of recombinant human IL-11 in mouse models of hepatic disease ${ }^{31-35}$. These findings led to a clinical trial using rhlL-11 in patients suffering from hepatitis $\mathrm{C}$ but this study was not taken forward ${ }^{36}$. The suggested protective role of IL-11 was questioned by recent animal studies showing that species-matched IL-11 is a key player in liver fibrosis in NAFLD as well as in the development of severe inflammation in $\mathrm{NASH}^{13,18,37}$.

Widjaja and co-workers recently showed that species-matched IL-11 is hepatotoxic and induces reactive oxygen species (ROS)-dependent hepatocyte cell death via cJun N-terminal kinase (JNK) along with inhibition of liver regeneration ${ }^{18}$. The difference of these findings with the former literature, where a high dose of rhIL-11 was injected to rodents, may be explained by the fact that rhlL-11 binds to the mouse $\mathrm{IL}-11$ receptor, but it does not activate the same signaling pathways as endogenous murine $\mathrm{IL}-11$ does. Dong et al. recently showed that IL-11 modulates the hepatocytic metabolism and suggested a transition mechanism via the cis-pathway from NAFLD to NASH, conditions where IL-11 levels are elevated in the liver and serum ${ }^{13}$. In IIra1 knockout mice, restoration of $\mathrm{IL}-11$ cis-signaling in hepatocytes reestablished steatosis and inflammation ${ }^{13}$. The pro-inflammatory effect could be confirmed in our murine model of ALD, but most importantly, was also seen in patients with $\mathrm{AH}$. Compared to cirrhosis patients, the circulating IL-11 levels were much higher. These data strongly suggest an important role of the IL-11 pathway in pathogenesis of human $\mathrm{AH}$, which is consistent with the more recent literature on IL-11. 
In a mouse model of APAP induced liver damage i.e., Widjaja et al. showed that circulating IL-11 levels are markedly elevated and genetic or pharmacologic inhibition of IL-11 signaling reduced liver damage and promoted liver regeneration ${ }^{23}$. These findings are in line with elevated IL-11 concentrations as a pro-inflammatory marker in ALD and NASH. Notably, in NASH, genetic or antibody-induced inhibition of IL-11 signaling reduced inflammation ${ }^{13}$. These findings are also in accordance with our findings that support further the idea that IL-11 as a pro-inflammatory cytokine in murine ALD.

Our study has some limitations. While the National Institute on Alcohol Abuse and Alcoholism (NIAAA) mouse model provides excellent insight into liver inflammation, liver fibrosis cannot be sufficiently addressed in this mode ${ }^{22}$. We found a beneficial effect of IL-11 inhibition on liver steatosis and neutrophilic inflammation. We could consistently show effects of IL-11 inhibition on pro-inflammatory factors, but we did not specifically investigate effects on immune cells themselves or the effects of endotoxins on liver derived cytokines, chemokines and reactive oxygen species (ROS). In addition, our training and validation cohort were collected from the same region/hospital.

In conclusion, IL-11 plays a key role in inflammation and liver damage in our murine model of ALD and we showed elevated IL-11 levels in blood and liver tissue in human $\mathrm{AH}$ and liver cirrhosis of various etiologies, with a clear cut-off to predict outcome. We propose that inhibition of IL-11 signalling as a key factor for reducing inflammation and hepatotoxicity in alcoholic liver disease might also impact long term fibrosis development. Therefore, anti-IL-11RA therapy could be a promising therapeutic option in treating $\mathrm{AH}$ and preventing cirrhosis. 


\section{Abbrevations}

AH - alcoholic hepatitis, ALD - alcoholic liver disease, ALT - alanine aminotransferase, anti-IL11RA -Interleukin 11 receptor antagonist, AST - aspartate transaminase, ETOH - ethanol, HE - hematoxylin and eosin, HCC - hepatocellular carcinoma, HSC - hepatic stellate cells, IgG - Immunglobulin G, IHC - immunohistochemistry, IL - Interleukin, , JNK - c-Jun N-terminal kinase, MELD - Model of End stage Liver Disease, MIP macrophage inflammatory protein, NAFLD - non-alcoholic fatty liver disease, NASH non-alcoholic steatohepatitis, NIAAA - National Institute on Alcohol Abuse and Alcoholism, RA - receptor antibody, ROC - receiver operating characteristic, ROS reactive oxygen species, TNF- $\alpha$ - tumor necrosis factor- $\alpha$, TGF $\beta$ - transforming growth factor beta, wt - wildtype 


\section{References}

1. Collaborators, G.A. Alcohol use and burden for 195 countries and territories, 1990-2016: a systematic analysis for the Global Burden of Disease Study 2016. Lancet 392, 1015-1035 (2018).

2. Asrani, S.K., Devarbhavi, H., Eaton, J. \& Kamath, P.S. Burden of liver diseases in the world. J Hepatol 70, 151-171 (2019).

3. Avila, M.A., et al. Recent advances in alcohol-related liver disease (ALD): summary of a Gut round table meeting. Gut $\mathbf{6 9}, 764-780$ (2020).

4. Lang, S., et al. Changes in the fecal bacterial microbiota associated with disease severity in alcoholic hepatitis patients. Gut Microbes 12, 1785251 (2020).

5. Parlesak, A., Schäfer, C., Schütz, T., Bode, J.C. \& Bode, C. Increased intestinal permeability to macromolecules and endotoxemia in patients with chronic alcohol abuse in different stages of alcohol-induced liver disease. J Hepatol 32, 742-747 (2000).

6. Tilg, H. \& Diehl, A.M. Cytokines in alcoholic and nonalcoholic steatohepatitis. N Engl J Med 343, 1467-1476 (2000).

7. Lopetuso, L.R., et al. Harmful Effects and Potential Benefits of Anti-Tumor Necrosis Factor (TNF)- $\alpha$ on the Liver. Int J Mol Sci 19(2018).

8. Schmidt-Arras, D. \& Rose-John, S. IL-6 pathway in the liver: From physiopathology to therapy. J Hepatol 64, 1403-1415 (2016).

9. Barbier, L., et al. Interleukin-1 Family Cytokines: Keystones in Liver Inflammatory Diseases. Front Immunol 10, 2014 (2019).

10. Yang, Y.C. Interleukin-11 (IL-11) and its receptor: biology and potential clinical applications in thrombocytopenic states. Cancer Treat Res 80, 321-340 (1995).

11. Taga, T. The signal transducer gp130 is shared by interleukin-6 family of haematopoietic and neurotrophic cytokines. Ann Med 29, 63-72 (1997).

12. Cook, S.A. \& Schafer, S. Hiding in Plain Sight: Interleukin-11 Emerges as a Master Regulator of Fibrosis, Tissue Integrity, and Stromal Inflammation. Annu Rev Med 71, 263-276 (2020).

13. Dong, J., et al. Hepatocyte-specific IL11 cis-signaling drives lipotoxicity and underlies the transition from NAFLD to NASH. Nat Commun 12, 66 (2021).

14. Metcalfe, R.D., Putoczki, T.L. \& Griffin, M.D.W. Structural Understanding of Interleukin 6 Family Cytokine Signaling and Targeted Therapies: Focus on Interleukin 11. Front Immunol 11, 1424 (2020).

15. Nguyen, P.M., Abdirahman, S.M. \& Putoczki, T.L. Emerging roles for Interleukin-11 in disease. Growth Factors 37, 1-11 (2019).

16. Schafer, S., et al. IL-11 is a crucial determinant of cardiovascular fibrosis. Nature $\mathbf{5 5 2}, \mathbf{1 1 0 - 1 1 5}$ (2017).

17. Ng, B., et al. Interleukin-11 is a therapeutic target in idiopathic pulmonary fibrosis. Sci Transl Med 11(2019).

18. Widjaja, A.A., et al. Inhibiting Interleukin 11 Signaling Reduces Hepatocyte Death and Liver Fibrosis, Inflammation, and Steatosis in Mouse Models of Nonalcoholic Steatohepatitis. Gastroenterology 157, 777-792.e714 (2019).

19. Adami, E., et al. IL11 is elevated in systemic sclerosis and IL11-dependent ERK signaling underlies TGF $\beta$-mediated activation of dermal fibroblasts. Rheumatology (Oxford) (2021).

20. easloffice@easloffice.eu, E.A.f.t.S.o.t.L.E.a. \& Liver, E.A.f.t.S.o.t. EASL Clinical Practice Guidelines for the management of patients with decompensated cirrhosis. J Hepatol 69, 406460 (2018).

21. Crabb, D.W., et al. Standard Definitions and Common Data Elements for Clinical Trials in Patients With Alcoholic Hepatitis: Recommendation From the NIAAA Alcoholic Hepatitis Consortia. Gastroenterology 150, 785-790 (2016).

22. Bertola, A., Mathews, S., Ki, S.H., Wang, H. \& Gao, B. Mouse model of chronic and binge ethanol feeding (the NIAAA model). Nat Protoc 8, 627-637 (2013). 
23. Widjaja, A.A., et al. Redefining IL11 as a regeneration-limiting hepatotoxin and therapeutic target in acetaminophen-induced liver injury. Sci Transl Med 13(2021).

24. Percie du Sert, N., et al. Reporting animal research: Explanation and elaboration for the ARRIVE guidelines 2.0. PLOS Biol 18, e3000411 (2020).

25. Galun, E. \& Rose-John, S. The regenerative activity of interleukin-6. Methods Mol Biol 982, 59-77 (2013).

26. Gao, B. \& Bataller, R. Alcoholic liver disease: pathogenesis and new therapeutic targets. Gastroenterology 141, 1572-1585 (2011).

27. Mandrekar, P., Bataller, R., Tsukamoto, H. \& Gao, B. Alcoholic hepatitis: Translational approaches to develop targeted therapies. Hepatology 64, 1343-1355 (2016).

28. Seitz, H.K., et al. Alcoholic liver disease. Nat Rev Dis Primers 4, 16 (2018).

29. Louvet, A. \& Mathurin, P. Alcoholic liver disease: mechanisms of injury and targeted treatment. Nat Rev Gastroenterol Hepatol 12, 231-242 (2015).

30. Newman, J.C. \& Verdin, E. $\beta$-hydroxybutyrate: much more than a metabolite. Diabetes Res Clin Pract 106, 173-181 (2014).

31. Nishina, T., et al. Interleukin-11 links oxidative stress and compensatory proliferation. Sci Signal 5, ra5 (2012).

32. Maeshima, K., et al. A protective role of interleukin 11 on hepatic injury in acute endotoxemia. Shock 21, 134-138 (2004).

33. Zhu, M., et al. IL-11 Attenuates Liver Ischemia/Reperfusion Injury (IRI) through STAT3 Signaling Pathway in Mice. PLoS One 10, e0126296 (2015).

34. Trepicchio, W.L., Bozza, M., Bouchard, P. \& Dorner, A.J. Protective effect of rhIL-11 in a murine model of acetaminophen-induced hepatotoxicity. Toxicol Pathol 29, 242-249 (2001).

35. Bozza, M., et al. Interleukin-11 reduces T-cell-dependent experimental liver injury in mice. Hepatology 30, 1441-1447 (1999).

36. Lawitz, E.J., Hepburn, M.J. \& Casey, T.J. A pilot study of interleukin-11 in subjects with chronic hepatitis $\mathrm{C}$ and advanced liver disease nonresponsive to antiviral therapy. Am J Gastroenterol 99, 2359-2364 (2004).

37. Widjaja, A.A., Chothani, S.P. \& Cook, S.A. Different roles of interleukin 6 and interleukin 11 in the liver: implications for therapy. Hum Vaccin Immunother 16, 2357-2362 (2020). 


\section{Tables}

Table 1: Patient's characteristics of the training cohort

\begin{tabular}{|c|c|c|c|c|}
\hline & $\begin{array}{l}\text { Healthy } \\
\qquad(n=19)\end{array}$ & $\begin{array}{r}\text { compensated } \\
\text { cirrhosis } \\
(n=79)\end{array}$ & $\begin{array}{l}\text { decompensated } \\
\text { cirrhosis }(n=31)\end{array}$ & $\begin{array}{r}\text { alcoholic } \\
\text { hepatitis } \\
(n=50)\end{array}$ \\
\hline Age (years) & $61( \pm 13.1)$ & $63.5( \pm 9.7)$ & $57(1 \pm 2.7)$ & $66( \pm 18.1)$ \\
\hline $\begin{array}{l}\text { Sex (female } \\
\%)\end{array}$ & 7 (36.8\%) & $29(36 \%)$ & $12(38 \%)$ & $17(34 \%)$ \\
\hline Weight (kg) & $75.5( \pm 18.1)$ & $76.4( \pm 17.7)$ & $76.3( \pm 15.9)$ & $79.8( \pm 17.6)$ \\
\hline Height (cm) & $173.3( \pm 8.3)$ & $173.2( \pm 9.4)$ & $173.5( \pm 11.1)$ & $173.3( \pm 7.0)$ \\
\hline BMI (kg/m2) & $28.2( \pm 3.6)$ & $26.3( \pm 4.8)$ & $25.9( \pm 5.5)$ & $26.3( \pm 3.3)$ \\
\hline AFLD & $\mathrm{nA}$ & 42 & 16 & 50 \\
\hline NAFLD & $\mathrm{nA}$ & 26 & 9 & $\mathrm{nA}$ \\
\hline
\end{tabular}




\begin{tabular}{|c|c|c|c|c|}
\hline Hepatitis B & $\mathrm{nA}$ & 5 & 1 & $\mathrm{nA}$ \\
\hline Hepatitis C & $\mathrm{nA}$ & 4 & 3 & $n A$ \\
\hline PBC/PSC/AIH & $n A$ & 2 & 2 & $\mathrm{nA}$ \\
\hline MELD & $\mathrm{nA}$ & $10.8( \pm 5.7)$ & $12.7( \pm 3.3)$ & $19.2( \pm 5.5)$ \\
\hline $\begin{array}{l}\text { Leucocytes } \\
(\mathrm{g} / \mathrm{l})\end{array}$ & $7.2( \pm 1.6)$ & $5.0( \pm 2.3)$ & $5.9( \pm 2.5)$ & $5.8( \pm 2.8)$ \\
\hline $\begin{array}{l}\text { Hemoglobin } \\
(\mathrm{g} / \mathrm{l})\end{array}$ & $140.5( \pm 16.3)$ & $123.0( \pm 22.6)$ & $122.2( \pm 21.3)$ & $109.0( \pm 20.4)$ \\
\hline $\begin{array}{l}\text { Hematocrit } \\
(\%)\end{array}$ & $0.4( \pm 0.05)$ & $0.35( \pm 0.06)$ & $0.35( \pm 0.05)$ & $0.31( \pm 0.06)$ \\
\hline $\begin{array}{l}\text { Platelet count } \\
(\mathrm{g} / \mathrm{l})\end{array}$ & $272.9( \pm 66.4)$ & $128.8( \pm 80.4)$ & $100.3( \pm 51.5)$ & $79.5( \pm 61.8)$ \\
\hline Urea (mg/dl) & $34.2( \pm 16.3)$ & $33.4( \pm 17.8)$ & $43.9( \pm 24.1)$ & $59.4( \pm 31.3)$ \\
\hline $\begin{array}{l}\text { Creatinine } \\
\text { (mg/dl) }\end{array}$ & $0.9( \pm 0.1)$ & $0.8( \pm 0.4)$ & $1.2( \pm 0.9)$ & $1.2( \pm 0.8)$ \\
\hline $\begin{array}{l}\text { Filtration rate } \\
(\mathrm{ml} / \mathrm{min} / \mathrm{m} 2)\end{array}$ & $58.2( \pm 9.2)$ & $57.0( \pm 10.3)$ & $55.4( \pm 8.4)$ & $56.4( \pm 9.1)$ \\
\hline $\begin{array}{l}\text { Bilirubin } \\
(\mathrm{mg} / \mathrm{dl})\end{array}$ & $0.8( \pm 0.4)$ & $1.4( \pm 0.7)$ & $4.2( \pm 2.6)$ & $5.7( \pm 2.3)$ \\
\hline $\begin{array}{l}\text { Sodium } \\
\text { (mmol/l) }\end{array}$ & $140.4( \pm 2.7)$ & $138( \pm 4.2)$ & $137.0( \pm 5.8)$ & $135.1( \pm 5.8)$ \\
\hline AST (U/I) & $36.6( \pm 13.2)$ & $60.7( \pm 31.2)$ & $88.5( \pm 39.9)$ & $98.2( \pm 41.4)$ \\
\hline ALT (U/I) & $29.1( \pm 17.2)$ & $44.9( \pm 28.0)$ & $71.0( \pm 58.2)$ & $89.1( \pm 28.5)$ \\
\hline $\begin{array}{l}\text { Gamma-GT } \\
(\mathrm{U} / \mathrm{I})\end{array}$ & $63.5( \pm 34)$ & $101.0( \pm 59.5)$ & $182.3( \pm 52.8)$ & $222.5( \pm 55.6)$ \\
\hline
\end{tabular}




\begin{tabular}{|l|r|r|r|r|}
\hline Alkaline & & & & \\
Phosphatase & & & & \\
\hline LDH (U/I) & $179.6( \pm 33.8)$ & $143.0( \pm 47.8)$ & $220.2( \pm 150.5)$ & $145.0( \pm 85.0)$ \\
\hline CRP (mg/dl) & $0.5( \pm 0.2)$ & $244.3( \pm 99.0)$ & $260.0( \pm 132.1)$ & $240.1( \pm 63.8)$ \\
\hline Quick (\%) & $99.0( \pm 13.3)$ & $67.3( \pm 17.3)$ & $55.9( \pm 22.6)$ & $54.5( \pm 15.8)$ \\
\hline INR & $1.0( \pm 0.1)$ & $1.3( \pm 0.2)$ & $1.4( \pm 0.5)$ & $1.5( \pm 0.4)$ \\
\hline PT (sec) & $30.5( \pm 2.6)$ & $38.7( \pm 9.1)$ & $46.1( \pm 17.3)$ & $43.2( \pm 18.4)$ \\
\hline Fibrinogen & $1.8( \pm 0.4)$ & $7.4( \pm 4.2)$ & $6.9( \pm 3.8)$ & $7.4( \pm 3.4)$ \\
\hline (mg/dl) & $334.0( \pm 53.5)$ & $274.4( \pm 106.8)$ & $212.9( \pm 144.5)$ & $204.6( \pm 83.1)$ \\
\hline Albumin & 4170.7 & 3362.7 & & 3014.8 \\
(mg/dl) & $( \pm 399.9)$ & $( \pm 607.4)$ & $3090.8( \pm 591.4)$ & $( \pm 476.2)$ \\
\hline Alpha-1- & & & & \\
\hline Fetoprotein & & & & \\
\hline
\end{tabular}

Data are expressed as case numbers (percentage) or mean \pm standard deviation. $\mathrm{kg}$ - kilograms, $\mathrm{cm}$ - centimeter, $\mathrm{kg} / \mathrm{m} 2$ - kilograms/square meter, AFLD - alcoholic fatty liver disease, NAFLD - nonalcoholic fatty liver disease, PBC - primary biliary cholangitis, PSC - primary sclerosing cholangitis, AlH - autoimmune hepatitis, mg/dl - milligrams/decliter, G/l - grams/liter, mmol/l - millimole/liter, $\mathrm{ml} / \mathrm{min} / \mathrm{m} 2$ milliliters/minute/square meter, $\mathrm{U} / \mathrm{I}$ - Units/liter, sec - second, $\mu \mathrm{g} / \mathrm{I}$ - microgram/liter Table 2: Multivariate COX Regression

\begin{tabular}{|l|l|l|l|l|l|l|l|l|l|}
\hline & \multicolumn{4}{|l|}{ MELD } & \multicolumn{4}{l|}{ IL-11 (pg/ml) } \\
\hline months & & 6 & 12 & 18 & 24 & 6 & 12 & 18 & 24 \\
\hline
\end{tabular}




\begin{tabular}{|l|l|l|l|l|l|l|l|l|l|}
\hline HR & & 0.95 & 0.56 & 2.31 & 0.97 & 0.82 & 0.61 & 1.87 & 1.37 \\
\hline $\mathbf{9 5 \%} \mathbf{C l}$ & & $0.84-$ & $0.41-$ & $1.04-$ & $0.69-$ & $0.62-$ & $0.39-$ & $1.04-$ & $0.87-$ \\
& & 1.06 & 0.72 & 5.07 & 1.32 & 1.03 & 0.95 & 3.23 & 1.98 \\
\hline $\mathbf{p - V a l u e}$ & & $<$ & $<$ & $<$ & $<$ & $<$ & $<$ & $<$ & $<$ \\
& & 0.05 & 0.05 & 0.05 & 0.03 & 0.04 & 0.05 & 0.04 & 0.05 \\
\hline
\end{tabular}

Multivariate Cox Regression: Laboratory parameters that were significantly predictive in univariate analysis and were not represented in MELD were included in a multivariate Cox-Regression with stepwise backwards selection. Of the multivariate analysis, only significant parameters are shown in the table. Abbrevations: MELD model of end stage liver disease, IL-11 - Interleukin 11, pg/ml - picograms/mililiter, $\mathrm{HR}$ - hazard ration, $\mathrm{Cl}$ - confidence interval

\section{Figure legends}

Fig.1: Severity of ALD correlates with circulating IL-11 levels and its hepatic expression

Study design and patient's selection (A). IL-11 serum levels and MELD score correlation in patients suffering from cirrhosis and alcoholic hepatitis and healthy controls (MELD in healthy controls was assumed 0) (B) IL-11 levels correlate with cirrhosis and alcoholic hepatitis in patients compared to healthy controls (MELD in healthy controls was assumed 0) (C). IL-11 levels distinguish between patients with compensated or decompensated cirrhosis, alcoholic hepatitis and healthy controls. (MELD in healthy controls was assumed 0) (D). Representative HE staining and IL-11 staining in liver tissue in healthy controls, patients suffering from cirrhosis and patients 
suffering from alcoholic hepatitis (E). Statistical analysis of IL-11 positive cells in liver tissue $(\mathrm{F}) .\left({ }^{*} p<0.05,{ }^{* *} p<0.01,{ }^{* * *} p<0.001\right)$

Abbreviations: IL-11 - Interleukin 11, MELD - model of end stage liver disease, pg/ml - picograms/milliliters, $\mathrm{H}$ - healthy control, $\mathrm{Cl}$ - cirrhosis, $\mathrm{CCl}$ - compensated cirrhosis, $\mathrm{DCl}$ - decompensated cirrhosis, $\mathrm{AH}$ - alcoholic hepatitis, $\mu \mathrm{m}$ - micrometer, $\mathrm{HE}-$ hematoxylin and eosinophils.

\section{Fig. 2: Serum IL-11 concentration predicts patient outcome}

AUROCs of the scores for prediction of transplant free survival (A). Kaplan Meyer curve for transplant-free survival with IL-11 cutoff $6.4 \mathrm{pg} / \mathrm{ml}$ (B). Kaplan Meier curve for validation cohort $(\mathrm{C})$. IL-11=1: Group 1 with serum IL-11 concentration $<6.4 \mathrm{pg} / \mathrm{ml}$. IL11=2: Group 2 with serum IL-11 concentration > $6.4 \mathrm{pg} / \mathrm{ml}$.

Abbreviations in order of their appearance: ALD - alcoholic liver disease, IL-11 Interleukin 11, MELD - model of end stage liver disease, $\mathrm{pg} / \mathrm{ml}$ - picograms/milliliters.

\section{Fig. 3: Inhibition of IL-11 signaling protects against experimental ALD}

Schematic timeline of the procedure. C57BL/6J female mice were pair-fed or EtOHfed for 15 days. The EtOH- group received either IgG or anti-IL11RA intraperitoneally (A). Application of anti-IL11RA resulted in significant lower ALT levels in the EtOH group compared to the IgG group (B). EtOH fed mice treated with anti-IL11RA showed decreased liver-to-body ratio (C), decreased weight loss (D) and decreased hepatic triglyceride accumulation compared to $\mathrm{EtOH}$ fed mice treated with $\lg \mathrm{G}(\mathrm{E}) .\left({ }^{*} p<0.05\right.$, ${ }^{* *} p<0.01,{ }^{* * *} p<0.001, \mathrm{n} \geq 5$ /group)

Abbreviations in order of their appearance: anti-IL11RA - Interleukin 11 Receptor Antibody, EtOH - Ethanol, IgG - Immunoglobulin G, ALT - alanine transferase, IL-11 - Interleukin 11 
Fig. 4: Inhibition of IL-11 signaling protects against ethanol-induced liver inflammation

Representative IL-11 staining images from all 3 groups (A). Administration of IL-11RA tend to reduce IL-11 in liver tissue $(B)$. Effect of IL-11RA administration on hepatic expression of proinflammatory cytokines in EtOH fed mice: TNF- $\alpha$ (C), TIMP1 (D), IL$10(\mathrm{E}), \mathrm{CXCL}-1(\mathrm{~F}), \mathrm{IL}-1 \mathrm{~b}(\mathrm{G}), \operatorname{MIP} 2(\mathrm{H})$ and IL-6 (I). $\left({ }^{*} p<0.05,{ }^{* *} p<0.01,{ }^{* * *} p<0.001\right.$, $n \geq 5$ /group)

Abbreviations in order of their appearance: IL-11R- Interleukin-11 receptor, IL-11 Interleukin 11, anti-IL11RA - Interleukin 11 Receptor Antibody, EtOH - Ethanol, IgG Immunoglobulin G, TIMP1-metallopeptidase inhibitor 1, IL-10 - Interleukin-10, TNFa tumor necrosis factor - $\alpha, \mathrm{CXCL}-1$ - chemokine (C-X-C motif) ligand $1, \mathrm{IL}-1 \beta-$ interleukin 1 beta, MIP 2 - macrophage inflammatory protein 2

Fig. 5: Anti-IL11RA treatment reduces infiltration of pro-inflammatory cells into the liver

Representative H\&E staining images from all 3 groups (A). Hepatic steatosis score decreased significantly in EtOH-fed wt mice treated with IL-11RA (B). Representative images and quantification of $\mathrm{MPO}^{+}$cells per high power field in the liver based on hepatic MPO immunoreactivity (D). Hepatic infiltration of $\mathrm{MPO}^{+}$neutrophils are significantly decreased in EtOH-fed mice treated with anti-IL11RA (E). Representative images and quantification of F4/80 positive macrophages per high power field in the liver based on hepatic F4/80 immunoreactivity (D). Hepatic infiltration of F4/80 macrophages are significantly decreased in EtOH-fed mice treated with anti-IL11RA (F). $n \geq 5$ /group.

Abbrevations in order of their appearance: anti-IL11RA - Interleukin 11 Receptor Antibody, IgG - Immunoglobulin G, EtOH - Ethanol, HPF - high power field, H\&E hematoxylin and eosin, MPO+ - myeloperoxidase positive 

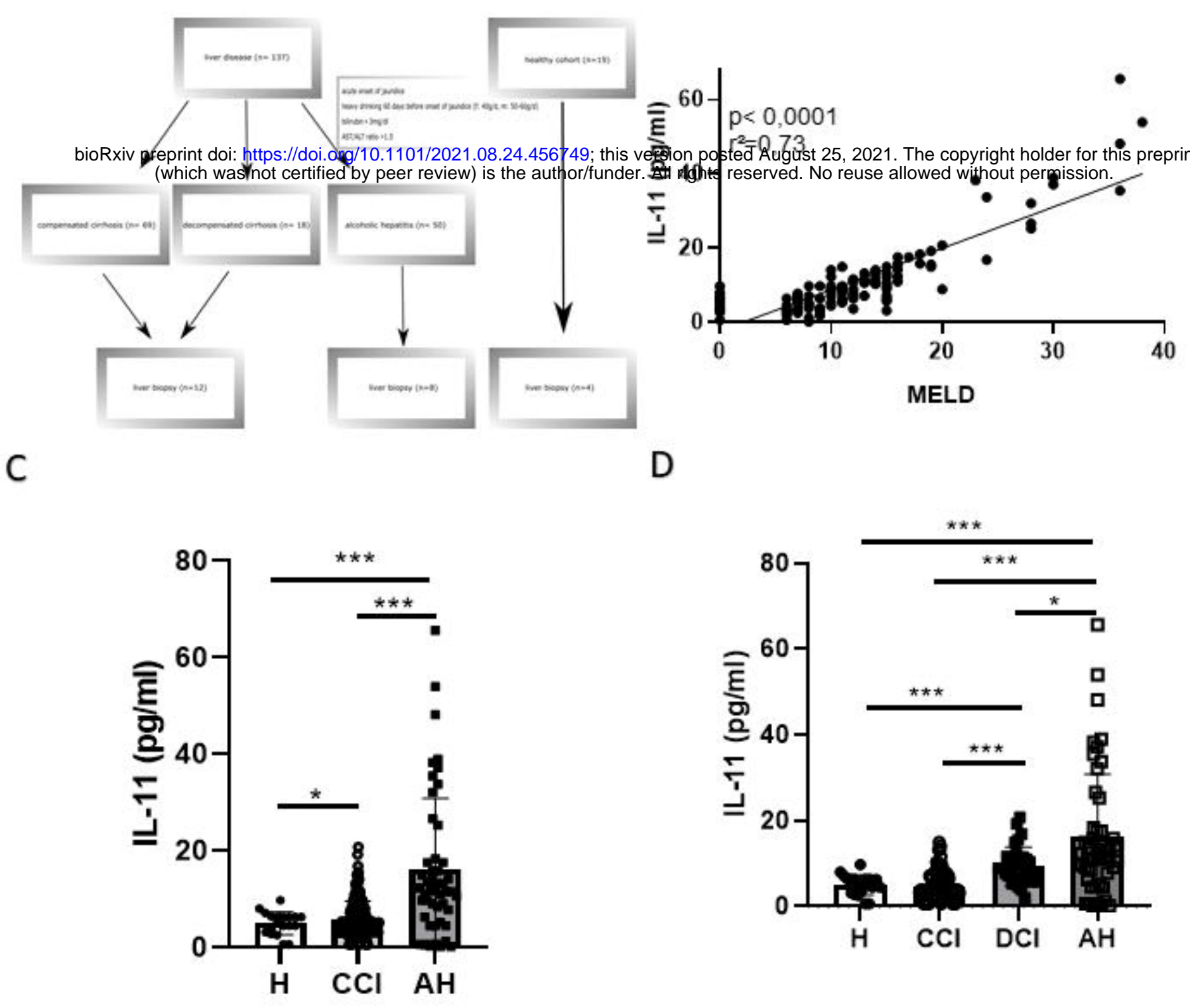

E

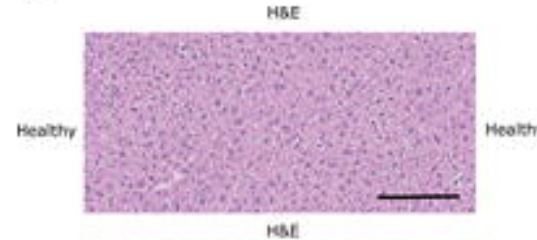

1est

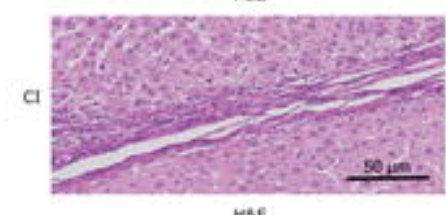

nst

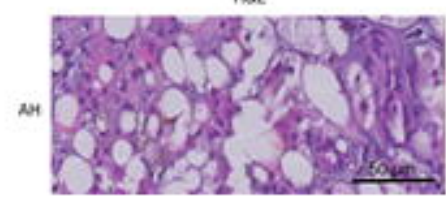

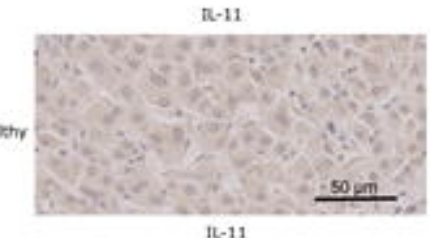

It-1
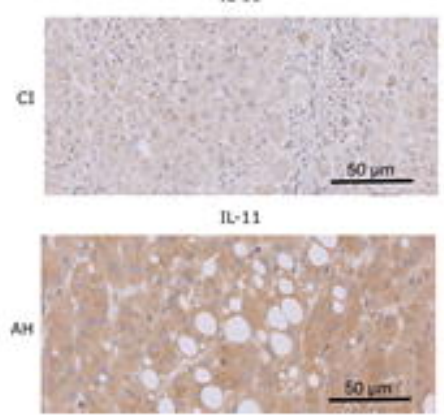

F

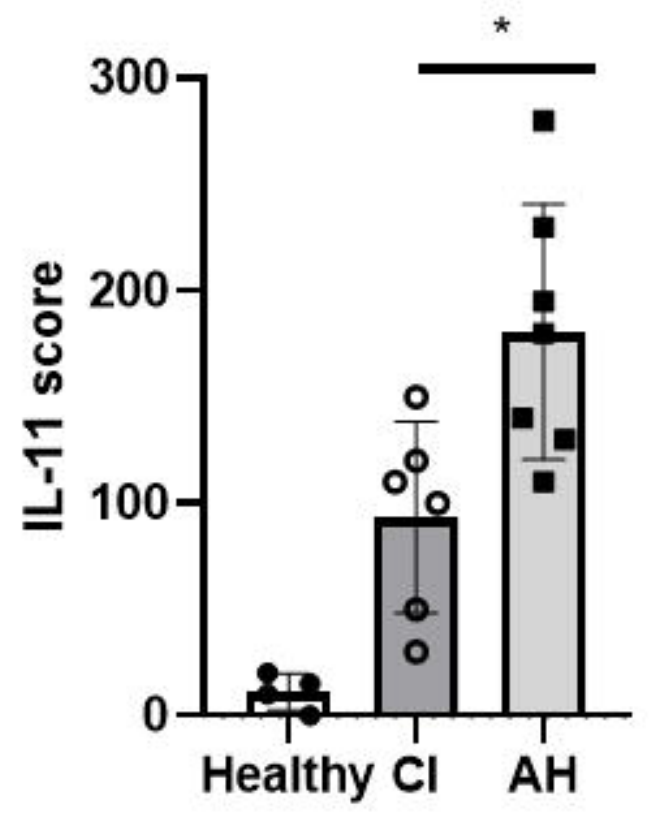


A

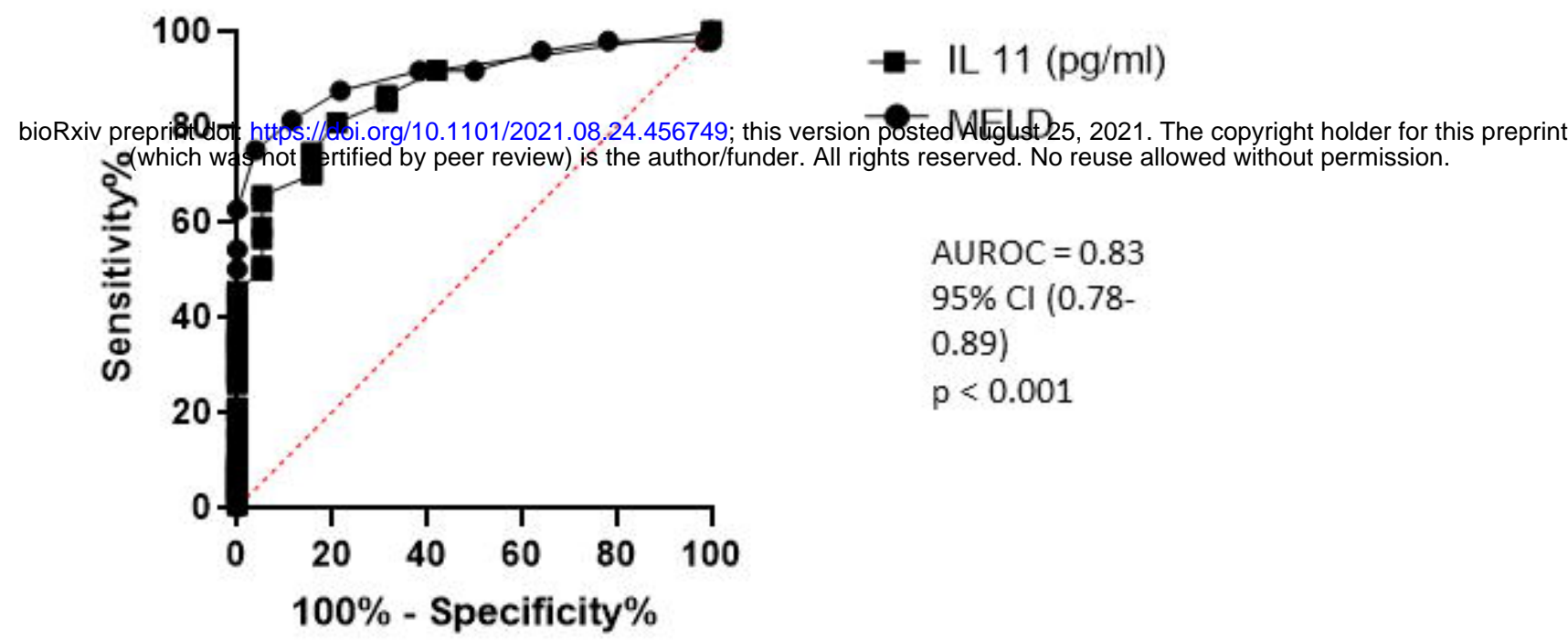

B

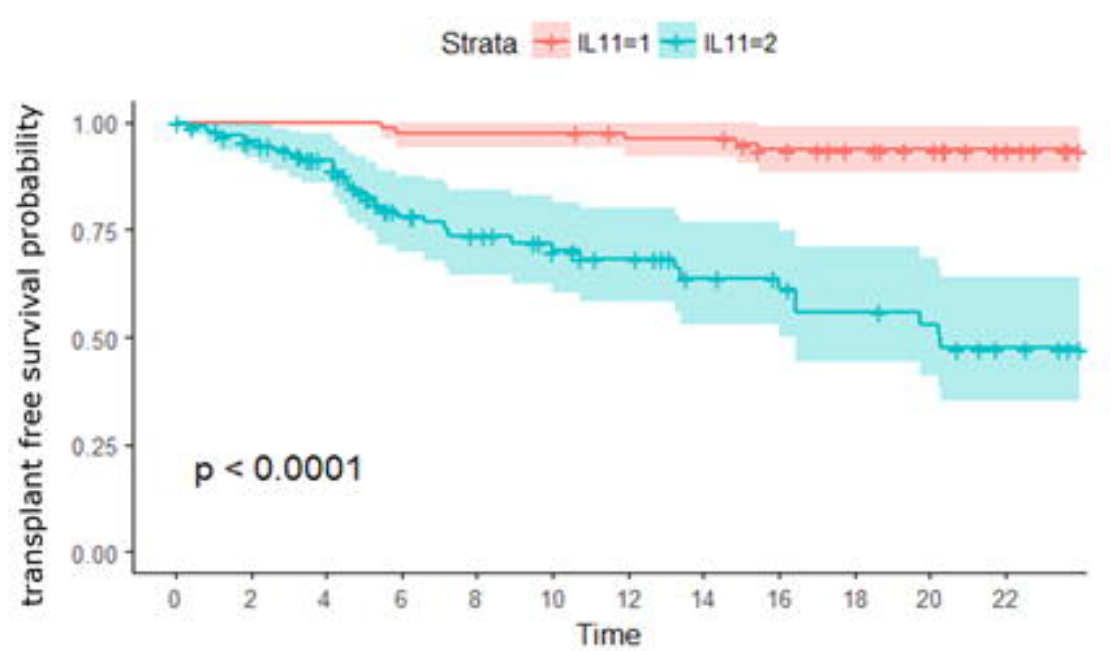

Number at risk

$\begin{array}{lllllllllllll}\text { UL } 11=1 & 81 & 81 & 81 & 79 & 79 & 79 & 76 & 76 & 71 & 67 & 64 & 59 \\ \text { L } 11=2 & 98 & 88 & 75 & 53 & 47 & 39 & 34 & 27 & 25 & 21 & 19 & 14\end{array}$

C

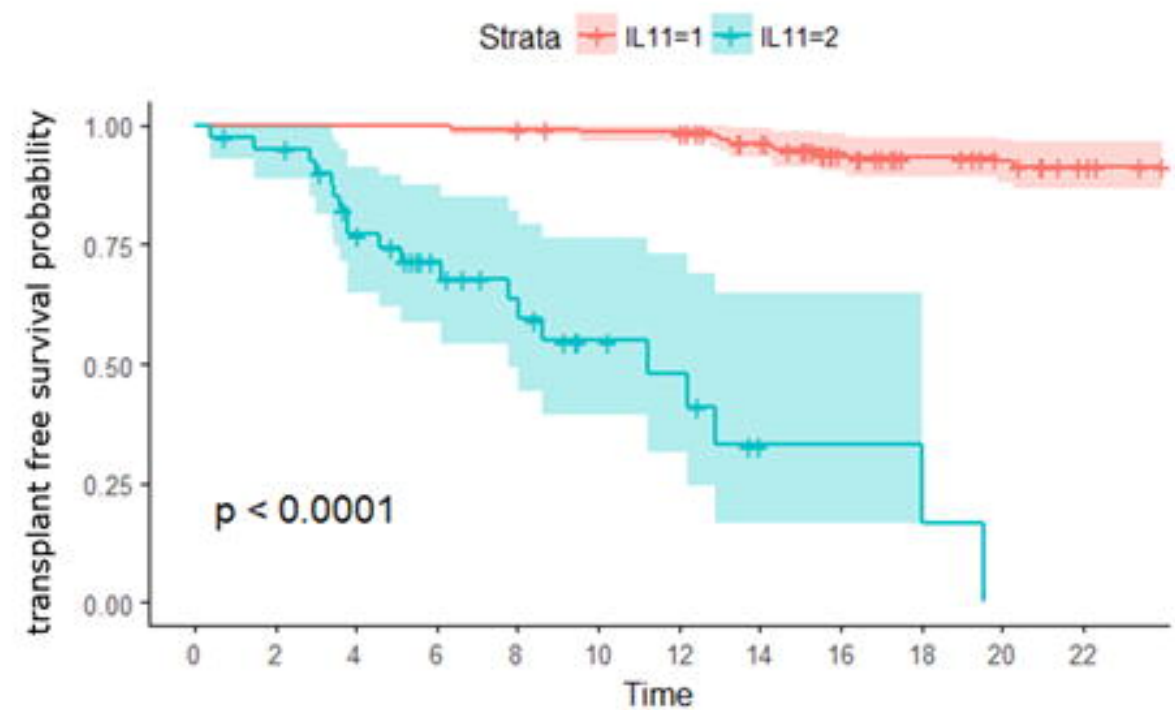

Number at risk
$\begin{array}{lllllllllllll}\text { IL } 11=1 & 144 & 144 & 144 & 144 & 143 & 140 & 140 & 131 & 114 & 106 & 101 & 95\end{array}$

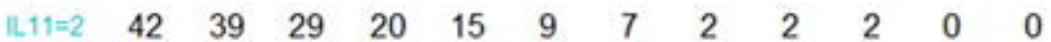


A

B

$50 \mathrm{ym}$

IgG

bioRxivpreprint doi: https://doi.org/10.1101/2021.08.24.456749; this versiond (which was not certified by peer review) is the author/funder. All rights reserved. No reus alld wed without permission.

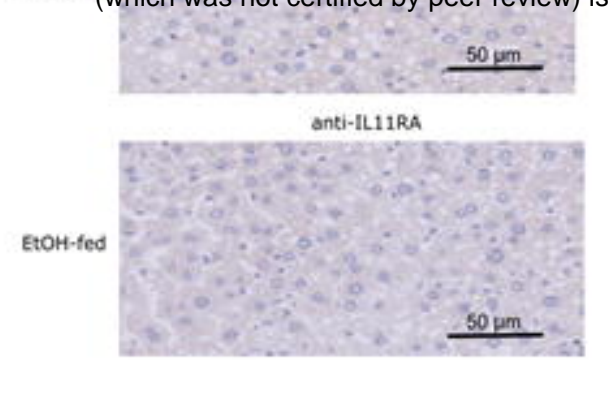

C
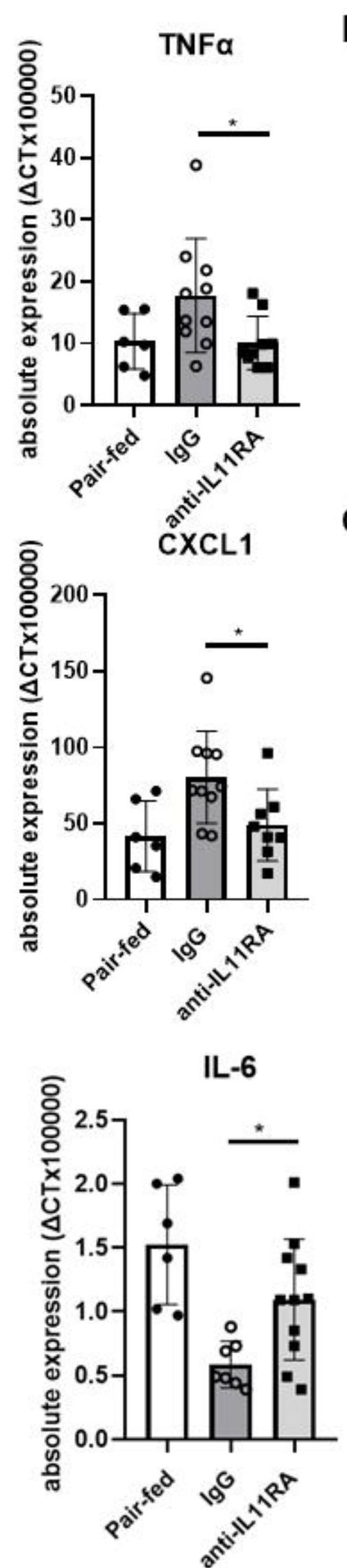

D $-\quad$ TIMP1 E

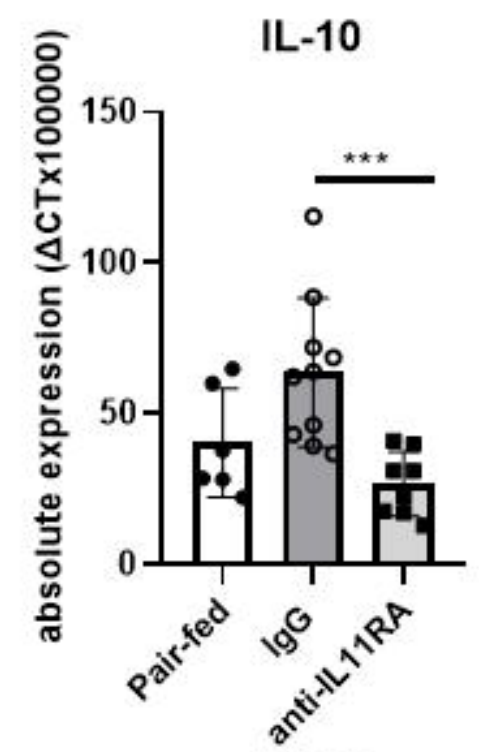

H

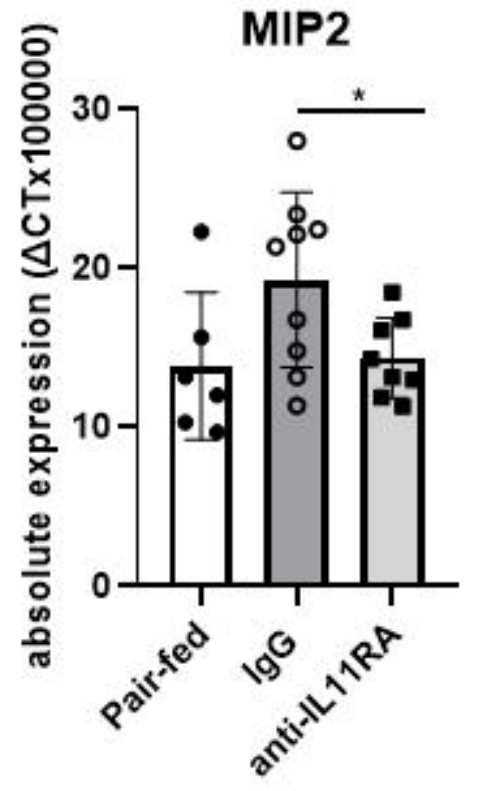


A

B

$50 \mathrm{ym}$

IgG

bioRxivpreprint doi: https://doi.org/10.1101/2021.08.24.456749; this versiond (which was not certified by peer review) is the author/funder. All rights reserved. No reus alld wed without permission.

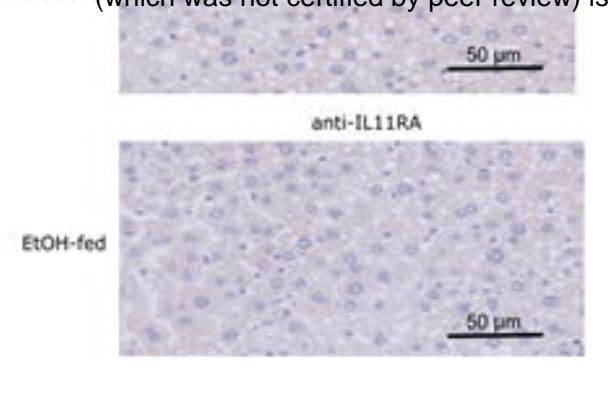

C
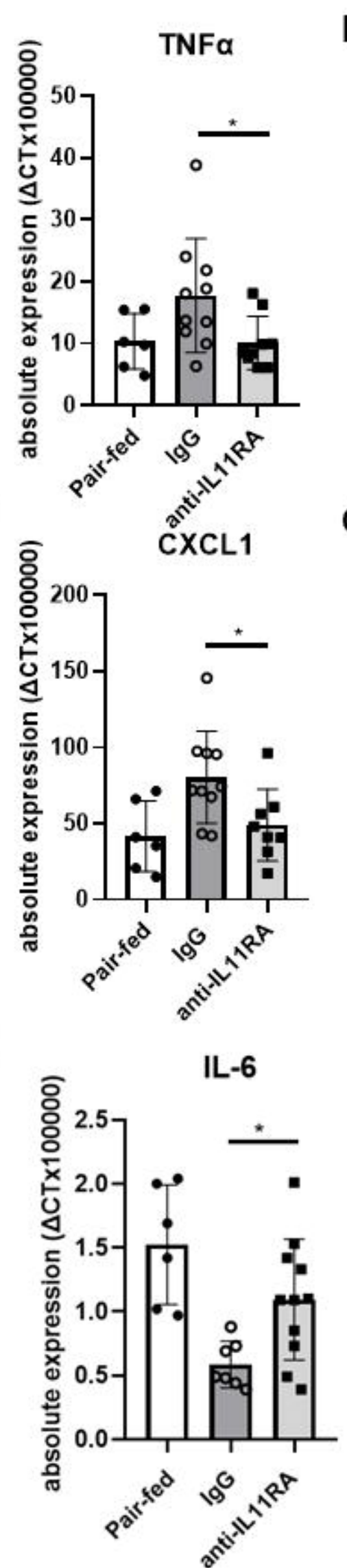

D $-\quad$ TIMP1 E

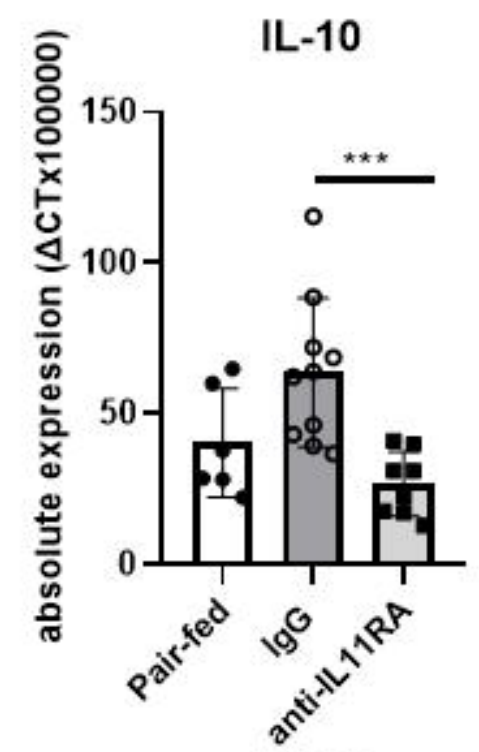

H

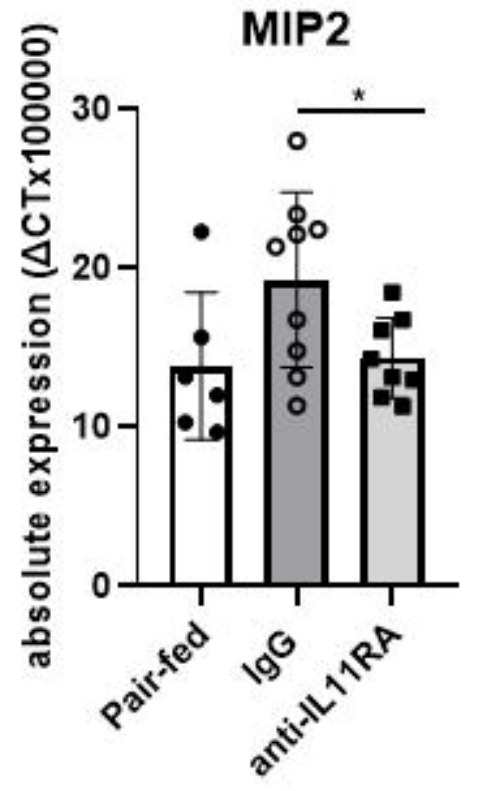


A

Pair-fed

EtoH-fed

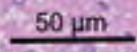

anti-IL11RA

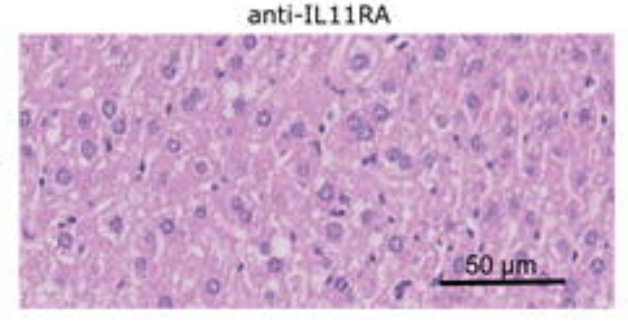

C

Pair-fed

$50 \mu \mathrm{m}$

IgG

EtoH-fed

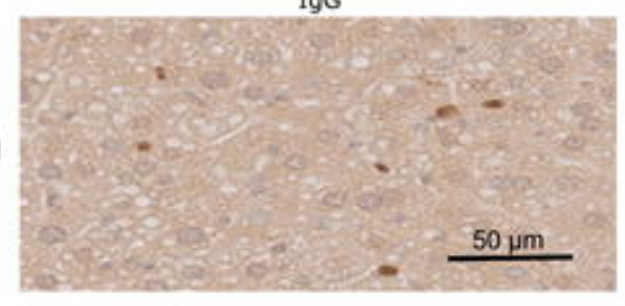

anti-IL11RA

EtoH-fed

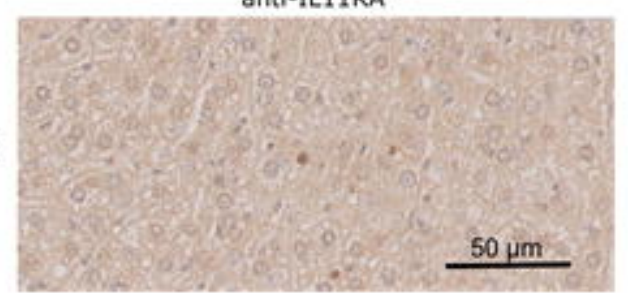

E

Pair-fed

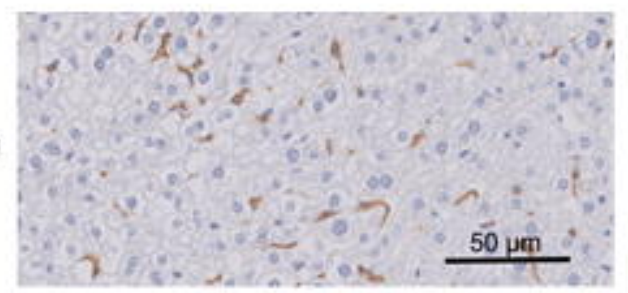

IgG

EtoH-fed

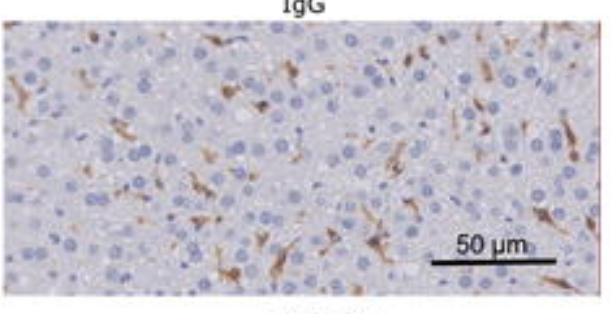

anti-IL11RA

EtoH-fed

$50 \mu \mathrm{m}$
B
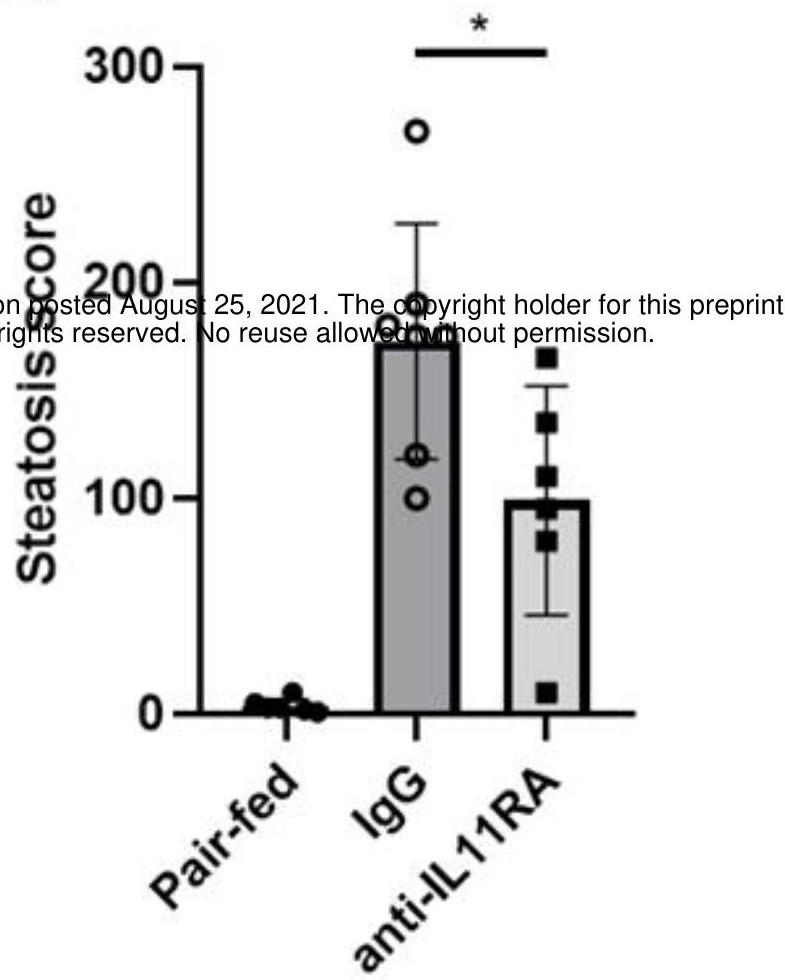

D

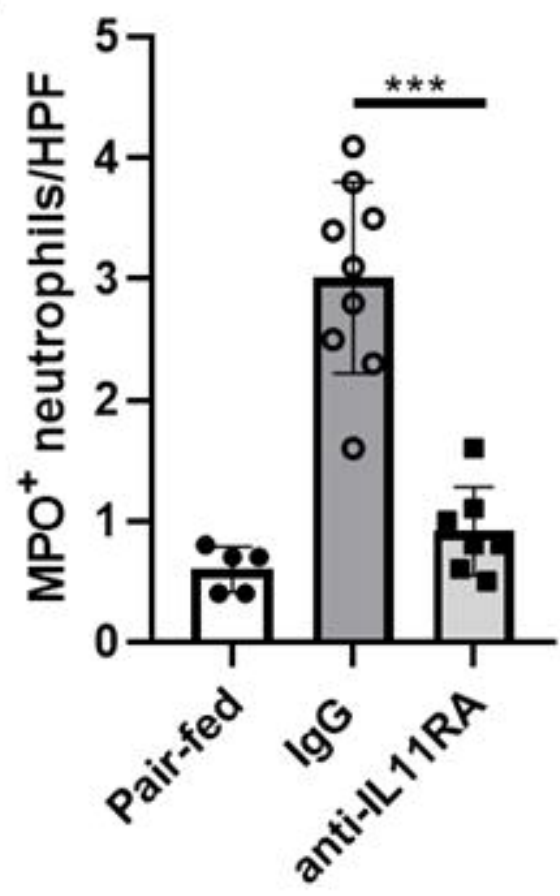

F

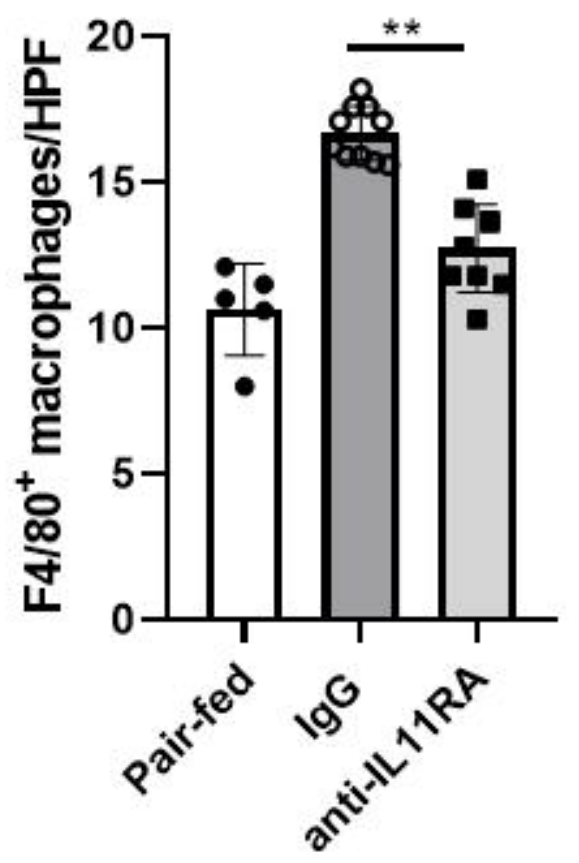

